\title{
Forecasting the Volatility of the Dow Jones Islamic Stock Market Index: Long Memory vs. Regime Switching*
}

\author{
Adnen Ben Nasr ${ }^{\dagger}$ Thomas Lux, ${ }^{\ddagger}$ Ahdi Noomen Ajmi ${ }^{\S}$ Rangan Gupta $~$
}

\begin{abstract}
The financial crisis has fueled interest in alternatives to traditional asset classes that might be less affected by large market gyrations and, thus, provide for a less volatile development of a portfolio. One attempt at selecting stocks that are less prone to extreme risks, is obeyance of Islamic Sharia rules. In this light, we investigate the statistical properties of the DJIM index and explore its volatility dynamics using a number of up-to-date statistical models allowing for long memory and regime-switching dynamics. We find that the DJIM shares all stylized facts of traditional asset classes, and estimation results and forecasting performance for various volatility models are also in line with prevalent findings in the literature. Overall, the relatively new Markov-switching multifractal model performs best under the majority of time horizons and loss criteria. Long memory GARCH-type models always improve upon the short-memory GARCH specification and additionally allowing for regime changes can further improve their performance.
\end{abstract}

JEL Classification: G15, G17, G23

Keywords: islamic finance, volatility dynamics, long memory, multifractals.

${ }^{*}$ Thomas Lux gratefully aknowledges financial support from the Spanish Ministry of Science and Innovation (ECO2011-23634), from Universitat Jaume I (P1.1B2012-27), and from the European Union 7th Framework Programme, under grant agreement no. 612955

${ }^{\dagger}$ BESTMOD, Institut Supérieur de Gestion de Tunis, Université de Tunis, Tunisia. Email: adnen.bennasr@isg.rnu.tn

${ }^{\ddagger}$ Department of Economics, University of Kiel, Germany \& Banco de España Chair in Computational Economics, University Jaume I, Castellon, Spain.

${ }^{\S}$ College of Sciences and Humanities in Slayel, Salman bin Abdulaziz University, Kingdom of Saudi Arabia. Email: ajmi.ahdi.noomen@gmail.com

${ }^{\top}$ Department of Economics, University of Pretoria, South Africa. Email: rangan.gupta@up.ac.za 


\section{Introduction}

The recent global financial crisis has exerted enormous negative impacts on conventional institutions and markets. Hence, a need has been felt for exploring alternatives to conventional financial practices that allow to reduce investment risks, increase returns, enhance financial stability, and reassure investors and financial markets. Given this, following the crisis, one has observed a renewed interest in Islamic finance, ${ }^{1}$ based on Sharia rules, as an approach that might offer products and instruments driven by greater social responsibility, ethical and moral values, and sustainability, and hence, may be better safeguarded against financial crises.

Against this backdrop, in this paper, we aim to model and forecast conditional volatility of the returns of the Dow Jones Islamic Market World Index (DJIM), accounting for both the possibility of long memory and structural changes in the volatility process. The choice of the DJIM is justified by the fact that it is the most widely used, and most comprehensive representative time series for the Islamic stock market (Hammoudeh et al., 2013). Note that, appropriate modeling and forecasting of volatility is of importance due to several reasons: Firstly, when volatility is interpreted as uncertainty, it becomes a key input to investment decisions and portfolio choices. Secondly, volatility is the most important variable in the pricing of derivative securities. To price an option, one needs reliable estimates of the volatility of the underlying assets. Thirdly, financial risk management according to the Basle Accord as established in 1996 also requires modeling and forecasting of volatility as a compulsory input to risk-management for financial institutions around the world. Finally, financial market volatility, as witnessed

\footnotetext{
${ }^{1}$ Assets in the Islamic industry have grown by $500 \%$ in the last five years and reached 1.6 trillion U.S. dollars in 2013 (Hammoudeh et al., 2013).
} 
during the recent "Great Recession" for the returns on DJIM like many other assets (see Figure 1), can have wide repercussions on the economy as a whole, via its effect on real economic activity and public confidence. Hence, estimates of market volatility can serve as a measure for the vulnerability of financial markets and the economy, and can help policy makers design appropriate policies. Evidently, appropriate modeling and accurate forecasting of the process of volatility has ample implications for portfolio selection, the pricing of derivative securities and risk management. While there is a rich literature on volatility modelling of 'conventional financial assets', not much evidence exists to date with respect to the Islamic stock market. We try to fill part of this gap using some of the most advanced tools available in contemporaneous econometric literature.

A large body of recent research suggests that there is significant evidence of long memory in the conditional volatility of various financial and economic time series (Ding et al., 1993; Baillie et al., 1996; Andersen and Bollerslev, 1997; Bollerslev and Mikkelsen, 1996; Lobato and Savin, 1998; Davidson, 2004). Another strand of research shows that there is also evidence for the occurrence of structural changes in the volatility process (Bos et al., 1999; Andreou and Ghysels, 2002; Rapach and Strauss, 2008; Rapach et al., 2008). In light of these two features (long memory and structural breaks), a body of research has suggested that both long memory and structural changes simultaneously characterize the structure of financial returns volatility (Lobato and Savin, 1998; Beine and Laurent, 2000; Morana and Beltratti, 2004; Martens et al., 2004; Baillie and Morana, 2007).

Motivated by this line of research that suggests co-existence of both long memory and structural change in the volatility processes of financial market data, following Ben Nasr et al., (2010), we estimate a model for the DJIM 
returns that allows the volatility of the returns to share such behavior. The idea is to allow the parameters in the conditional variance equation of a Fractionally Integrated Generalized Autoregressive Conditional Heteroskedasticity (FIGARCH) model to be time dependent. More precisely, the change of the parameters is assumed to evolve smoothly over time using a logistic smooth transition function, to yield a so-called Fractionally Integrated Time Varying Generalized Autoregressive Conditional Heteroskedasticity (FITVGARCH) model.

Further, a related line of research on long memory and structural changes in volatility discusses the connection between these phenomena. In fact, volatility persistence may be due to switching of regimes in the volatility process, as first suggested by Diebold (1986) and Lamoureux and Lastrapes (1990). This literature concludes that it could be very difficult to distinguish between true and spurious long memory processes. This ambiguity motivates us to include a new type of Markov-switching model in addition to our array of volatility models (i.e., GARCH, FIGARCH, FITVGARCH) - the Markov-switching multifractal (MSM) model of Calvet and Fisher (2001). Despite allowing for a large number of regimes, this model is more parsimonious in parameterization than other regime-switching models. It is furthermore well-known to give rise to apparent long memory over a bounded interval of lags (Calvet and Fisher, 2004) and it has limiting cases in which it converges to a 'true' long memory process. To the best of our knowledge, this is the first attempt in forecasting the volatility process for the DJIM returns using a wide variety of advanced volatility models trying to capture long-memory, structural breaks and the fact that structural breaks can lead to the spurious impression of long-memory. The rest of the paper is organized as follows: Section 2 provides basic information on GARCH, 
FIGARCH, FITVGARCH and MSM models, while Section 3 presents the data and the empirical results. Finally, Section 4 concludes.

\section{GARCH, FIGARCH, FITVGARCH and MSM Volatility models}

Univariate models of volatility usually consider the following specification of financial returns measured over equally spaced discrete points in time $t=1, \ldots, T$ :

$$
y_{t}=\mu_{t}+\sigma_{t} u_{t}
$$

where $y_{t}=p_{t}-p_{t-1}$ with $p_{t}=\ln P_{t}$ the logarithmic asset price, $\mu_{t}=$ $E\left[y_{t} \mid \mathcal{F}_{t-1}\right]$ and $\sigma_{t}^{2}=\operatorname{Var}\left[y_{t} \mid \mathcal{F}_{t-1}\right]$ the conditional mean and the conditional variance (volatility), respectively. The information set $\mathcal{F}_{t-1}$ is assumed to contain all relevant information up to period $t-1$. Moreover, $u_{t}$ is an independently and identically distributed disturbance with mean zero and variance one. Although $u_{t}$ can be drawn from various stationary distributions, in this study we let $u_{t} \sim N(0,1)$. The return components $\mu_{t}$ and $\sigma_{t}$ can be specified according to the assumed data generating process. For the purpose of this study we use the simple specification $\mu_{t}=\mu+\rho y_{t-1}$. Defining $r_{t}=y_{t}-\mu_{t}$, the 'centered' returns can be modeled as

$$
r_{t}=\sigma_{t} u_{t}
$$

Now we turn to volatility modelling. Returns in financial markets are typically found to be heteroskedastic with high autocorrelation of all measures of volatility (e.g., squared or absolute returns). To capture this feature, 
the literature had developed the time-honored class of models with autoregressive conditional heteroskedasticity. As the benchmark version of this class of models, the GARCH(1,1) model of Bollerslev (1986) assumes that the volatility dynamics is governed by

$$
\sigma_{t}^{2}=\omega+\alpha r_{t-1}^{2}+\beta \sigma_{t-1}^{2}
$$

where the restrictions on the parameters are $\omega>0, \alpha, \beta \geq 0$ and $\alpha+\beta<1$.

The FIGARCH model introduced by Baillie et al. (1996) expands the GARCH variance equation by considering fractional differences. As in the case of the GARCH model, we restrict our attention to one lag in both the autoregressive term and in the moving average term. The $\operatorname{FIGARCH}(1, d, 1)$ model is, then, given by

$$
\sigma_{t}^{2}=\omega+\left[1-\beta L-(1-\delta L)(1-L)^{d}\right] r_{t}^{2}+\beta \sigma_{t-1}^{2}
$$

where $L$ is the lag operator, $d$ is the parameter of fractional differentiation and the restrictions on the parameters are $\beta-d \leq \delta \leq(2-d) / 3$ and $d\left(\delta-2^{-1}(1-d)\right) \leq \beta(d-\beta+\delta)$. In the case of $d=0$, the FIGARCH model reduces to the standard $\operatorname{GARCH}(1,1)$ model. For $0<d<1$ the binomial expansion of the fractional difference operator introduces an infinite number of past lags with hyperbolically decaying coefficients. Note that in practice, the infinite number of lags in the FIGARCH model with $0<d<1$ must be truncated. We employ a lag truncation of 1000 steps.

The FITVGARCH model introduced by Ben Nasr et al. (2010) expands the FIGARCH model of Baillie et al. (1996) by allowing the conditional variance parameters to change over time. The FITVGARCH $(p, d, q)$ model 
is given by:

$$
\left[1-\Phi_{t}(L)\right](1-L)^{d} u_{t}^{2}=\omega_{t}+\left[1-\beta_{t}(L)\right] v_{t}
$$

where $v_{t}=u_{t}^{2}-\sigma_{t}^{2}, \omega_{t}=\omega_{1}+\omega_{2} F\left(t^{*} ; \gamma, c\right), \Phi_{t}(L)=\Phi_{1}(L)+\Phi_{2}(L) F\left(t^{*} ; \gamma, c\right)$; $\Phi_{1}(L)=\phi_{1,1} L+\ldots+\phi_{1, q} L^{q}, \Phi_{2}(L)=\phi_{2,1} L+\ldots+\phi_{2, q} L^{q}, \beta_{t}(L)=\beta_{1}(L)+$ $\beta_{2}(L) F\left(t^{*} ; \gamma, c\right) ; \beta_{1}(L)=\beta_{1,1} L+\ldots+\beta_{1, p} L^{p}, \beta_{2}(L)=\beta_{2,1} L+\ldots+\beta_{2, p} L^{p}$, $F\left(t^{*} ; \gamma, c\right)$ is a logistic smooth transition function defined as

$$
F\left(t^{*} ; \gamma, c\right)=\left(1+\exp \left\{-\gamma \prod_{k=1}^{K}\left(t^{*}-c_{k}\right)\right\}\right)^{-1}
$$

with constraints $\gamma>0$ and $c_{1} \leq c_{2} \leq \ldots \leq c_{K}$ for the transition points in the standardized time variable $t^{*}=t / T$ with $T$ as the sample size. The transition function $F\left(t^{*} ; \gamma, c\right)$ is a continuous function bounded between 0 and 1. The parameter $\gamma$ corresponds to the speed of transition between the two regimes, while the parameter $c_{k}$, known as the threshold parameter, indicates when, within the range of $t$, the transitions take place.

The roots of the polynomials $\left[1-\Phi_{t}(L)\right]$ and $\left[1-\beta_{t}(L)\right]$ should again be outside the unit circle for all $t$. This implies that $\left[1-\Phi_{t}(1)\right]>0$ and $\left[1-\beta_{t}(1)\right]>0$, if we restrict our setting to one lag like under GARCH and FIGARCH. With $K=1$, the parameters of the FITVGARCH model change smoothly over time from $\left(\omega_{1}, \phi_{1, i}, \beta_{1, j}\right)$ to $\left(\omega_{1}+\omega_{2}, \phi_{1, i}+\phi_{2, i}, \beta_{1, j}+\beta_{2, j}\right), i=$ $1, \ldots, q, j=1, \ldots, p$. The transitions between regimes happen instantaneously when $t^{*}=c_{1}$ and $\gamma$ is large. When $\gamma \rightarrow 0$, the $\operatorname{FITVGARCH}(p, d, q)$ model in (5) and (6) nests the FIGARCH $(p, d, q)$ model of Baillie et al. (1996) since the logistic transition function becomes constant and equal to $1 / 2$. As with GARCH and FIGARCH, we only consider one lag in the volatility equation, i.e. we impose $\mathrm{p}=\mathrm{q}=1$.

Estimation of the GARCH, FIGARCH and FITVGARCH models can 
be done via the Quasi Maximum Likelihood (QML) method. The $l$-period ahead forecasts $\hat{\sigma}_{t+l \mid t}^{2}$ for these models can be obtained most easily by recursive substitution of one-step ahead forecasts $\hat{\sigma}_{t+1}^{2}$. Note that one obtains volatility forecasts from FITVGARCH in much the same way as for FIGARCH using the active regime at time t. The advantage of FITVGARCH would consist in detecting a possible regime switch within the in-sample used for estimation so that the set of parameters might be different from those of a FIGARCH model without regime switching both estimated for the same series.

We now turn to a description of the MSM model. An in-depth analysis of this model can be found in Calvet and Fischer (2004) and Lux (2008). In the MSM model, instantaneous volatility is determined by the product of $k$ volatility components or multipliers $M_{t}^{(1)}, M_{t}^{(2)}, \ldots, M_{t}^{(k)}$ and a scale factor $\sigma^{2}:$

$$
\sigma_{t}^{2}=\sigma^{2} \prod_{i=1}^{k} M_{t}^{(i)}
$$

Following the basic hierarchical principle of the multifractal approach, each volatility component $M_{t}^{(i)}$ will be renewed at time $t$ with a probability $\gamma_{i}$ depending on its rank within the hierarchy of multipliers, and will remain unchanged with probability $1-\gamma_{i}$. Convergence of the discrete-time MSM to a Poisson process in the continuous-time limit requires to formalize transition probabilities according to:

$$
\gamma_{i}=1-\left(1-\gamma_{k}\right)^{\left(b^{i-k}\right)}
$$

with $\gamma_{k}$ and $b$ parameters to be estimated (Calvet and Fisher, 2001). Since we are not interested in the continuous-time limit in this article, we fol- 
low Lux (2008) and use pre-specified transition probabilities $\gamma_{i}=2^{i-k}$ rather than the specification of eq. (8). The negligence of a more flexible parametrization of transition probabilities also can be motivated by the fact that the in-sample fit and out-of-sample forecasting performance of both alternatives laid out above have been found to be almost invariant compared to the influence of other (estimated) parameters (Lux, 2008). We consider different specifications of the MSM model varying $k$ from 2 through 15 and choose the one at which the objective function does not improve anymore by more than a very small difference. The consideration of a high number of multipliers $k$ can be motivated by previous findings that show that even levels beyond $k>10$ may improve the forecasting capabilities of the MSM for some series and proximity to temporal scaling of empirical data might be closer (Liu et al., 2007). Indeed, having 'too many' multipliers is always harmless as the other parameter estimates would remain unchanged beyond some threshold and 'superfluous' multipliers with very long life times would just absorb part of the scale parameter.

The MSM model is a Markov-switching process with $2^{k}$ states. The model is fully specified once we have determined the distribution of the volatility components. It is usually assumed that the multipliers $M_{t}^{(i)}$ follow either a Binomial or a Lognormal distribution. In the MSM framework, only one parameter has to be estimated for the distribution of volatility components, since one would normalize the distribution so that $E\left[M_{t}^{(i)}\right]=1$.

Here we use the Lognormal MSM (LMSM) model, in which multipliers are determined by random draws from a Lognormal distribution with parameters $\lambda$ and $\nu$, i.e.

$$
M_{t}^{(i)} \sim L N\left(-\lambda, \nu^{2}\right)
$$


Normalization via $E\left[M_{t}^{(i)}\right]=1$ leads to

$$
\exp \left(-\lambda+0.5 \nu^{2}\right)=1
$$

from which a restriction on the shape parameter $\nu$ can be inferred: $\nu=$ $\sqrt{2 \lambda}$. Hence, the distribution of volatility components corresponds to a one-parameter family of Lognormals with the normalization restricting the choice of the shape parameter. Thus, the LMSM parameters to be estimated are just $\lambda$ and $\sigma$ for all specifications $k=2, \ldots, 15$.

Lux (2008) has introduced a GMM estimator that is universally applicable to all possible specifications of MSM processes. In the GMM framework the unknown parameter vector $\varphi=(\lambda, \sigma)^{\prime}$ is obtained by minimizing the distance of empirical moments from their theoretical counterparts, i.e.

$$
\widehat{\varphi}_{T}=\arg \min _{\varphi \in \Phi} f_{T}(\varphi)^{\prime} A_{T} f_{T}(\varphi)
$$

with $\Phi$ the parameter space, $f_{T}(\varphi)$ the vector of differences between sample moments and analytical moments, and $A_{T}$ a positive definite and possibly random weighting matrix. Under standard regularity conditions that are routinely satisfied by MSM models, the GMM estimator $\widehat{\varphi}_{T}$ is consistent and asymptotically normal. ${ }^{2}$

In order to account for the proximity to long memory characterizing MSM models we follow Lux (2008) in using logarithmic differences of absolute returns together with the pertinent analytical moment conditions,

\footnotetext{
${ }^{2}$ The standard regularity conditions are problematic for the preceding 'first generation' multifractal model of Mandelbrot et al. (1997) because of its restrictions to a bounded time interval. This is not an issue for the 'second generation' MSM of Calvet and Fisher (2001) which by its very nature is a variant of a Markov-switching model.
} 
i.e.

$$
\xi_{t, T}=\ln \left|r_{t}\right|-\ln \left|r_{t-T}\right|
$$

Using (2) and (7) in (12) we get the expression

$$
\xi_{t, T}=0.5 \sum_{i=1}^{k}\left(m_{t}^{(i)}-m_{t-T}^{(i)}\right)+\ln \left|u_{t}\right|-\ln \left|u_{t-T}\right|
$$

where $m_{t}^{(i)}=\ln M_{t}^{(i)}$. The variable $\xi_{t, T}$ only has nonzero autocovariances over a limited number of lags. To exploit the temporal scaling properties of the MSM model, covariances of various orders $q$ over different time horizons are chosen as moment conditions, i.e.

$$
\operatorname{Mom}(T, q)=E\left[\xi_{t+T, T}^{q} \cdot \xi_{t, T}^{q}\right]
$$

for $q=1,2$ and $T=1,5,10,20$, together with $E\left[r_{t}^{2}\right]=\sigma^{2}$ for identification of $\sigma^{2}$.

Out-of-sample forecasting of the MSM model estimated via GMM is performed for the zero-mean time series $Y_{t}=r_{t}^{2}-\widehat{\sigma}^{2}$ for $l$-step ahead horizons, by means of best linear forecasts computed with the generalized LevinsonDurbin algorithm developed by Brockwell and Dahlhaus (2004).

\section{Empirical analysis}

In this section, we present the results of our empirical study, starting with the description of the data, the in-sample estimation results, and then proceed to the out-of-sample forecast comparison of the different volatility models discussed above. 


\subsection{Data}

The various volatility models are estimated using daily data of the Global Dow Jones Islamic Market World Index (DJIM). The DJIM index measures the performance of the global universe of investable equities that have been screened for Sharia compliance. The companies in this index pass the industry and financial ratio screens. The regional allocation for DJIM is classified as follows: $60.14 \%$ for the United States; $24.33 \%$ for Europe and South Africa; and $\mathbf{1 5 . 5 3 \%}$ for Asia (Hammoudeh et al., 2013). Our data spans the period of January 1, 1996 to September 2, 2013, implying a total of 5750 observations. Note that the start and end date for the index is governed purely by data availability at the time of writing this paper. The time series for the index is sourced from Bloomberg. In order to get a preliminary idea about the data set, we present, in Figure 1, the daily index in levels and returns. Note that daily returns are normalized by taking 100 times the first difference of the natural log of the index. Table 1 gives some basic statistics for the DJIM index returns. Inspecting the first four moments of the data (prior to normalization), we find pronounced negative skewness (probably due to the inclusion of the time of the financial crisis) and excess kurtosis as it typically characterizes financial time series.

The Jarque-Bera test rejects the hypothesis of Normally distributed returns at any level of significance. Similarly, conducting a series of Box-Ljung tests for different lags, independence of raw, squared and absolute returns is rejected at any traditional level of significance. In line with practically all other such analysis of financial time series, the Box-Ljung statistics is orders of magnitude larger for both squared and absolute returns than for the raw data. Hence, there is much higher persistence in the measures of volatility than in the raw returns themselves. It is this very feature that gave 
rise to the development of volatility models with autoregressive dependence and long memory. We finally also show the so-called Hill estimator for the tail index, i.e. the decay of the unconditional distribution of returns in its outer, extremal region. The literature unequivocally finds the extreme part of the distribution following a power-law, i.e. $\operatorname{Prob}\left(\left|\tau_{t}\right|>x\right) \sim x^{-\alpha}$, with the 'tail index' typically around 3 (which has been denominated the 'cubic' law of large returns), cf. Cont (2001). Applying the conditional maximum likelihood estimator of Hill (1975), we report results for the decay parameter $\alpha$ together with its 95 percent confidence intervals in Table 1. Tail index estimates for assumed extremal tail regions are in the vicinity of around 3 as it had been found for essentially all other financial series scrutinized in the literature. The DJIM data also displays the slight decrease of the estimate for larger tail sizes that is presumably due to more and more contamination of tail observations with those from the central part of the distribution.

So far, we do not find anything out of the ordinary. Returns from the DJIM seem to share all basic stylized facts of stock market data and more broadly defined classes of financial assets. In particular, the Box-Ljung test points to a very similar structure of the volatility dynamics (already visible in the clear clustering of volatility in Fig. 1), and the obeyance of the 'cubic law' of large returns indicates that Islamic stocks share the same risk structure like more conventional assets.

Fig.1 shows that daily returns are, in general, highly volatile, with volatility being highest during the month of October in 2008. Given this, we used the first 3926 (until December 29, 2006) observations for in-sample estimation, and the remaining 1824 observations for out-of-sample forecasting of the volatility of DJIM returns, with the out-of-sample period being chosen to coincide with the global financial crisis - a period of high volatility. 
Table 1: Time Series Statistics of DJIM Returns

\begin{tabular}{|c|c|c|}
\hline Mean: & 0.000 & \\
\hline Std. dev: & 0.010 & \\
\hline Skewness: & -0.367 & \\
\hline Kurtosis: & 8.889 & \\
\hline Bera-Jarque statistics: & $19055.047(0.000)$ & \\
\hline Box-Ljung statistics for: & Return $\quad$ Squared Return & Absolute Return \\
\hline Lag 8 & $\begin{array}{ll}91.039 & 3545.467 \\
(0.000) & (0.000)\end{array}$ & $\begin{array}{l}2657.314 \\
(0.000)\end{array}$ \\
\hline Lag 12 & $\begin{array}{ll}92.981 & 5318.485 \\
(0.000) & (0.000)\end{array}$ & $\begin{array}{l}3339.935 \\
(0.000)\end{array}$ \\
\hline Lag 16 & $\begin{array}{ll}103.141 & 6217.137 \\
(0.000) & (0.000)\end{array}$ & $\begin{array}{l}4691.161 \\
(0.000)\end{array}$ \\
\hline $\begin{array}{ll}\text { Hill tail index at } & 10 \% \text { tail } \\
& 5 \% \text { tail } \\
& 1 \% \text { tail }\end{array}$ & $\begin{array}{l}2.754(2.529-2.979) \\
3.133(2.770-3.495) \\
3.575(2.647-4.503)\end{array}$ & \\
\hline
\end{tabular}

Note: For the Bera-Jarque and Box-Ljung statistics, p-values are given in brackets; for the tail index estimates, the brackets contain the 95 percent confidence intervals of the point estimates based upon the limiting distribution of the estimator.

\subsection{Estimation}

We now turn to estimation of our four volatility models introduced above. In-sample estimation results of the different models are reported in Table 2. The results corresponding to GARCH-type models, i.e., $\operatorname{GARCH}(1,1)$, FIGARCH $(1, \mathrm{~d}, 1)$ and FITVGARCH $(1, \mathrm{~d}, 1)$ indicate that the constant parameter $(\hat{\omega})$ is significant at the $5 \%$ level. Also, we observe a statistically significant effect of past volatility on current volatility $(\hat{\beta})$ and of past squared innovations on current volatility $(\hat{\phi})$ in all three models at the $5 \%$ significance level. In addition, estimation results of both FIGARCH and FITVGARCH models indicate strong evidence of long memory in the conditional variance of the DJIM index returns, with statistically significant estimates of $d$, and almost the same value in both models $(\simeq 0.48)$. FITVGARCH estimation 

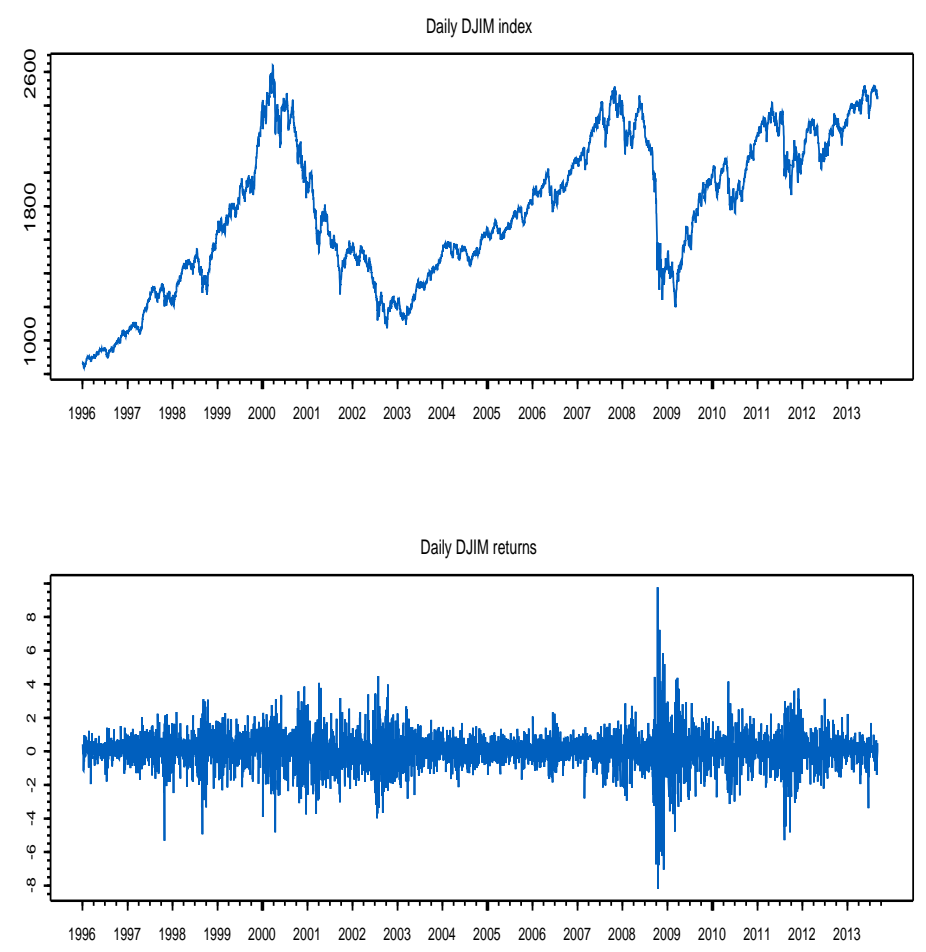

Figure 1: Daily DJIM index in level and returns

results show that the regime specific FIGARCH parameters experience significant changes. The estimated threshold parameter is significant at the $5 \%$ significance level and equal to 0.6114 , indicating that the change in the volatility structure took place at about the time point $\hat{t}=0.6114 \times T \simeq 2400$, corresponding to mid-August 2002. The high estimated value of the smoothness parameter $\gamma$ implies a sudden change in the parameters of the variance equation. We now turn to the estimation of the Lognormal MSM model. The in-sample selection procedure discussed above results in the choice of the number of multipliers to be $k=14$. Hence, it requires a high number of hierarchical levels to obtain saturation of the GMM objective function in eq. (11). The estimates of the Lognormal parameter $(\hat{\lambda})$ and the scale 
factor parameter $(\hat{\sigma})$ have values of 1.464 and 0.809 respectively.

\subsection{Out-of-sample analysis}

In this section, the out-of-sample forecasting accuracy of the considered volatility models is analyzed. Daily volatility forecasts were computed for the out-of-sample period; from January 2, 2007 to September 2, 2013. For each day in the forecasting period, forecasts are computed for horizons of various lengths: $1,5,10,20,60,70,80,90$ and 100 days. We have used the set of the in-sample parameter estimates and have kept it fixed over the outof-sample period, as it is computationally demanding and time consuming to estimate the FI(TV)GARCH models using maximum-likelihood. The $l$ steps-ahead forecast $\hat{\sigma}_{t+h \mid t}$ is obtained by appropriate substitution based on the conditional volatility specification and the forecast errors as given by:

$$
e_{t+h \mid t}=\epsilon_{t+h}^{2}-\hat{\sigma}_{t+h \mid t}^{2}
$$

For forecast evaluation, we use both the mean squared forecast error (MSE) and the mean absolute forecast error (MAE) criteria. The null hypothesis of equality of forecast performance from different models is tested in a pairwise comparison using the Diebold and Mariano (1995) (DM) test and the modified DM type test statistics for nested models of Clark and West (2007), depending on the models to be compared. Furthermore, we use the superior predictive ability (SPA) test introduced by Hansen (2005) that allows for the simultaneous test of $n$ similar null hypotheses against a group of alternatives.

Table 3 reports MSE and MAE of volatility forecasts for the four volatility models described previously relative to the MSE and MAE obtained with 
a naive forecast using the constant historical volatility (computed as average squared on absolute returns) of the in-sample period. A value $<1$ would, thus, indicate that the pertinent model improves upon historical volatility under the respective criterion. Based on the MSE criterion, the long memory model with time varying parameters, FITVGARCH, seems to be the best model for certain short-horizon volatility forecasts such as 1, 20 and 30 days, while FIGARCH turns out to be the best model at a horizon of 10 days. For longer horizons, 40 days and beyond, the MSM model is the best one, while FITVGARCH comes in second place. Also, we find that the simple GARCH model can not outperform the long memory-type GARCH models at any horizon. According to the MAE criterion, results are somewhat different: we find that the MSM model dominates over all the other models at all horizons. With respect to the GARCH-type models, we find that the FIGARCH model outperforms FITVGARCH only at horizons 1 and 5 days. For longer horizons, 10 days and beyond, the FITVGARCH model performs better than GARCH and FIGARCH models.

Comparison with historical volatility shows, however, that some of the time series models improve upon the naive forecast (HV) under the MAE criterion, while only the MSM model consistently outperform historical volatility at all time horizons. This difference in the performance under the MSE and MAE criterion indicates that time-series models are generally better suited to forecast large realisations of volatility than average sized ones (as the former have a higher influence on the average MSE compared to the average MAE). Similar results have been found for other time series before (e.g. Levey and Lux, 2012), and they appear to some extent plausible and even reassuring since it is the occurrence of large clusters of highly autocorrelated fluctuations that has motivated the development of modern asset 
pricing models like the ones used in this study.

Table 4 contains results of pairwise forecast comparison, for the four models, with the Diebold and Mariano (1995) test using both squared forecast error and absolute forecast error loss functions. For the cases of nested models, we apply the modified Diebold-Mariano test by Clark and West (2007). Note that in the hierarchy of GARCH type models the simpler ones are always nested in the more complex ones and historical volatility is nested in all time series models. In contrast MSM and any of the GARCH-type models are non-nested. We show results for the adjusted test only for the MSE criterion as it applies to quadratic loss criteria only by design. The results represent the $p$-values of the null hypothesis that forecast performance at horizon $l$ of model 1 is equal to the one of model 2 against the one-sided alternative that model 2's forecast performance is superior than the one of model 1. At $10 \%$ level of significance and in terms of the squared error loss function, the MSM model is outperformed by the other models at lower horizons $(l \leq 20)$ but it dominates when the forecast horizon exceeds 50 days. We also find that the FITVGARCH model seems to outperform the FIGARCH model for $l \geq 30$. In terms of the absolute error loss function, the MSM outperforms the other models at all horizons, while the FITVGARCH model outperforms the FIGARCH model for $l \geq 10$.

We also apply the SPA test of Hansen (2005) using the same two loss functions, MSE and MAE. Hansen's test allows to compare one model's performance to that of a whole set of competitors. The null hypothesis of the test is that a particular model (benchmark model) is not inferior to all the other candidate models. Table 5 presents the SPA test for each model including also historical volatility in the comparison. We find that, based on both MSE and MAE used as loss functions in the SPA test, the long-memory 
models FGARCH and FITVGARCH perform pretty similarly, where none of them can be significantly outperformed at any horizon not exceeding 40 days. But beyond this horizon, they can be outperformed by other models at least at the $10 \%$ level of significance. The MSM model, in contrast, can be outperformed at short-horizons ( $l \leq 20$ days) but not for longer horizons, $l \geq 30$.

Table 2: Estimated parameters of four models for DJIM daily index returns

\begin{tabular}{lllllllll}
\hline & \multicolumn{2}{l}{ GARCH } & \multicolumn{2}{l}{ FIGARCH } & MSM & \multicolumn{3}{c}{ FITVGARCH } \\
\hline$\hat{d}$ & - & - & 0.4831 & $(0.0627)$ & - & - & 0.4888 & $(0.0450)$ \\
$\hat{c}$ & - & - & - & - & - & - & 0.6114 & $(0.0278)$ \\
$\hat{\gamma}$ & - & - & - & - & - & - & 500 & $(3268.62)$ \\
$\hat{\omega} \mid \hat{\omega}_{1}$ & 0.0031 & $(0.0009)$ & 0.0089 & $(0.0042)$ & - & - & 0.0159 & $(0.0032)$ \\
$\hat{\phi}_{1} \mid \hat{\phi}_{1,1}$ & 0.0418 & $(0.0049)$ & 0.3186 & $(0.0526)$ & - & - & 0.3030 & $(0.0238)$ \\
$\hat{\beta}_{1} \mid \hat{\beta}_{1,1}$ & 0.9540 & $(0.0054)$ & 0.7467 & $(0.0377)$ & - & - & 0.7314 & $(0.0295)$ \\
$\hat{\omega}_{2}$ & - & - & - & - & - & - & -0.0139 & $(0.0034)$ \\
$\hat{\phi}_{2,1}$ & - & - & - & - & - & - & 0.0691 & $(0.0344)$ \\
$\hat{\beta}_{2,1}$ & - & - & - & - & - & - & 0.0771 & $(0.0169)$ \\
$\hat{\lambda}$ & - & - & - & - & 1.4639 & - & - & - \\
$\hat{\sigma}$ & - & - & - & - & 0.8089 & - & - & - \\
\hline \multicolumn{7}{l}{ Note: Standard errors are given in parentheses. } \\
\hline
\end{tabular}


Table 3: Forecast evaluation for DJIM return volatility based on MSE and MAE criteria

\begin{tabular}{|c|c|c|c|c|c|c|c|c|}
\hline & \multicolumn{2}{|c|}{ GARCH } & \multicolumn{2}{|c|}{ FIGARCH } & \multicolumn{2}{|l|}{ MSM } & \multicolumn{2}{|c|}{ FITVGARCH } \\
\hline Horizon & MSE & MAE & MSE & MAE & MSE & MAE & MSE & MAE \\
\hline 1 & 0.7976 & 1.0585 & 0.7874 & 1.0581 & 0.8643 & 1.0058 & 0.7752 & 1.0694 \\
\hline 5 & 0.8037 & 1.0636 & 0.7751 & 1.0569 & 0.9021 & 1.0015 & 0.7924 & 1.0583 \\
\hline 10 & 0.8577 & 1.0922 & 0.8368 & 1.0910 & 0.9389 & 1.0148 & 0.8406 & 1.0830 \\
\hline 20 & 0.9378 & 1.1427 & 0.9165 & 1.1404 & 0.9682 & 1.0248 & 0.9152 & 1.1287 \\
\hline 30 & 9986 & 1.1831 & 0.9682 & 1.1774 & 0.9811 & 1.0286 & 0.9657 & 1.1608 \\
\hline 40 & 1.0394 & 1.2006 & 1.0027 & 1.1969 & 0.9877 & 1.0302 & 0.9988 & 1.1776 \\
\hline 50 & 1.0503 & 1.2060 & 1.0135 & 1.2065 & 0.9906 & 1.0310 & 1.0087 & 1.1842 \\
\hline 60 & 1.0475 & 1.1988 & 1.0139 & 1.2055 & 0.9920 & 1.0306 & 1.0092 & 1.1807 \\
\hline 70 & 1.0422 & 1.1977 & 1.0130 & 1.2111 & 0.9931 & 1.0303 & 1.0083 & 1.1844 \\
\hline 80 & 1.0363 & 1.1908 & 1.0116 & 1.2114 & 0.9939 & 1.0301 & 1.0075 & 1.1844 \\
\hline 90 & 1.0340 & 1.1923 & 1.0126 & 1.2170 & 0.9948 & 1.0302 & 1.0087 & 1.1876 \\
\hline 100 & 1.0356 & 1.1940 & 1.0157 & 1.2217 & 0.9957 & 1.0300 & 1.0119 & 1.1911 \\
\hline
\end{tabular}

Note: MSE and MAE for all four models are displayed relative to the MSE and MAE of a constant forecast using historical volatility as estimated from the in-sample series. Entries in italics represent the best performing model for the pertinent loss function and forecasting horizon. 
Table 4: Diebold and Mariano test results

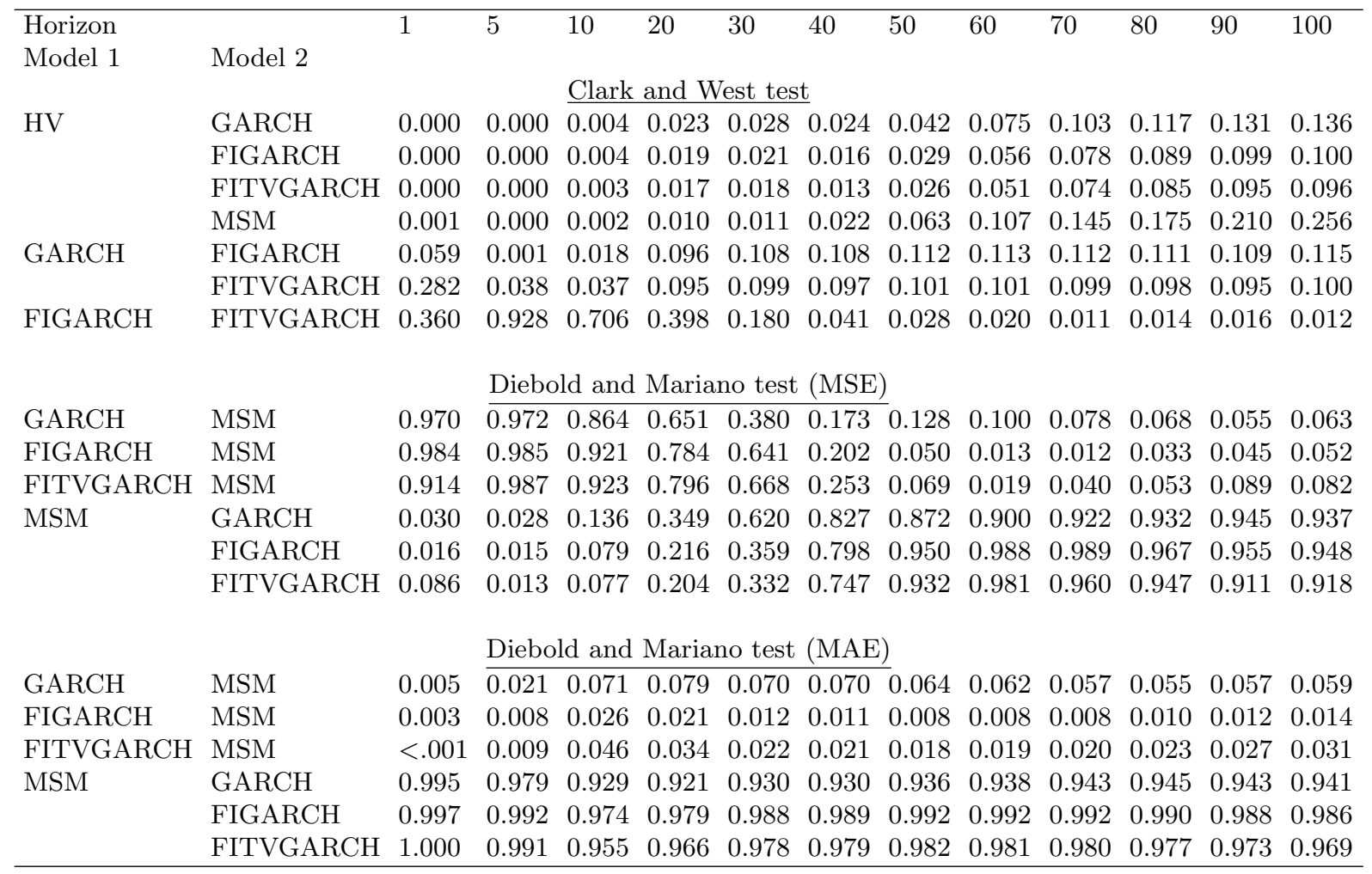

Note: Table entries represent the one-sided p-values of the Clark and West (2007) test and the Diebold and Mariano (1995) test (the second is based on both squared and absolute prediction errors). The Clark-West test is used in the case of nested models, while the Diebold-Mariano test is utilized for non-nested models. For both models, the null hypothesis is that the forecast performance at horizon $h$ of model 2 is equal to the one of model 1 against the one-sided alternative that model 2's forecast performance is superior to the one of model 1. 
Table 5: Superior predictive ability (SPA) test results

\begin{tabular}{|c|c|c|c|c|c|c|c|c|c|c|}
\hline \multirow[t]{2}{*}{ Horizon } & \multicolumn{5}{|c|}{ Squared errors } & \multicolumn{5}{|c|}{ Absolute errors } \\
\hline & $\overline{\mathrm{HV}}$ & GARCH & FIGARCH & MSM & FITVGARCH & $\overline{\mathrm{HV}}$ & GARCH & FIGARCH & MSM & FITVGARCH \\
\hline 1 & 0 & 0.2 & 0.625 & 0.048 & 0.728 & 0.680 & 0.048 & 0.013 & 0.320 & 0.005 \\
\hline 5 & 0 & 0.003 & 1 & 0.015 & 0.113 & 0.535 & 0.048 & 0.033 & 0.465 & 0.022 \\
\hline 10 & 0 & 0.003 & 0.708 & 0.013 & 0.292 & 1 & 0.018 & 0.003 & 0.020 & 0.005 \\
\hline 20 & 0 & 0.02 & 0.480 & 0.06 & 0.520 & 1 & 0 & 0 & 0 & 0 \\
\hline 30 & 0 & 0.003 & 0.590 & 0.308 & 0.870 & 1 & 0 & 0 & 0 & 0 \\
\hline 40 & 0 & 0.003 & 0.223 & 0.823 & 0.335 & 1 & 0 & 0 & 0 & 0 \\
\hline 50 & 0 & 0 & 0.010 & 1 & 0.050 & 1 & 0 & 0 & 0 & 0 \\
\hline 60 & 0 & 0.003 & 0.010 & 1 & 0.058 & 1 & 0 & 0 & 0 & 0 \\
\hline 70 & 0 & 0.003 & 0.015 & 1 & 0.090 & 1 & 0 & 0 & 0 & 0 \\
\hline 80 & 0 & 0 & 0.037 & 0.95 & 0.098 & 1 & 0 & 0 & 0 & 0 \\
\hline 90 & 0 & 0.007 & 0.030 & 1 & 0.095 & 1 & 0 & 0 & 0 & 0 \\
\hline 100 & 0 & 0.01 & 0.005 & 1 & 0.040 & 1 & 0 & 0 & 0 & 0 \\
\hline
\end{tabular}

Note: The table entries represent the p-values of the SPA test of Hansen (2005) using two loss functions (MSE and MAE). The null hypothesis is that a particular model (benchmark model) cannot be outperformed by other candidate models. Each column shows the outcome of this test in terms of the one-sided p-ratios for the pertinent model against all alternatives.

For the GARCH model, we find that it is inferior to alternative models under MSE at all horizons but $l=1$. Interestingly, historical volatility is also clearly out performed by some alternative forecasts so that the application of our battery of time series models adds value in term of forecasting accuracy under the MSE criterion. This is different under the MAE criterion where the null of non-inferiority of $\mathrm{HV}$ for the alternative forecasts is never rejected. If we would exclude HV from the competition, the SPA test would indicate superiority of MSM compared to the GARCH type model at all forecast horizons in line with the results of Tables 3 and 4 . However, under the MAE criterion, all our time series models would not add value to the naive approach of using the in-sample average on a predictor for future volatility. 


\section{Conclusions}

In the wake of the recent global financial crisis, a need has emerged for a reconsideration of many facets of the existing financial system. Among other developments, this has also led to a renewal of interest in Islamic finance. In essence, Islamic finance attempts to provide financial products and instruments that are consistent with certain principles such as social responsibility, ethical and moral values and sustainability. Given the prevalent interest in such products, we have investigated the statistical properties of the Dow Jones Islamic Market World Index (DJIM), and have applied up-todate volatility models to model and forecast conditional volatility of DJIM returns, accounting for both long memory and structural changes in the volatility process, as well as the fact that volatility persistence may be due to structural breaks. Given this, we use four different types of volatility models, namely, the Generalized Autoregressive Conditional Heteroskedasticity (GARCH), Fractionally Integrated Generalized Autoregressive Conditional Heteroskedasticity (FIGARCH), Fractionally Integrated Time Varying Generalized Autoregressive Conditional Heteroskedasticity (FITVGARCH) and Markov-switching multifractal (MSM) models. While the GARCH model serves as our benchmark volatility model, FIGARCH allows for long memory, FITVGARCH covers both long memory and structural breaks simultaneously, and the MSM model captures regime-switching that might lead to spurious time-series characteristics close to 'true' long memory. The choice of the DJIM is justified by the fact that it is the most widely used, most comprehensive representative, and has the most adequate time series for the Islamic stock market.

Our results show that the MSM model appears to be superior to other models considered, especially at longer horizons, and with absolute errors 
as loss criterion, for forecasting the volatility of the DJIM returns, and that it outperforms the GARCH, FIGARCH and FITVGARCH for most of the out-of-sample forecast comparison tests. However, this superiority against GARCH-type models only has economic value under the MSE criterion, while under the MAE loss function, all time-series models show predictive capabilities that are inferior to historical volatility. Another finding is that modeling the properties of long memory and time varying parameters in the volatility process, as in the FITVGARCH model, can improve the forecast performance for short and long forecasting horizons. Not surprisingly, the classical GARCH model seems to be the worst performing model in terms of forecasting future volatility among the models considered. All in all, these results are not too different from those of other previous studies of the comparative performance of volatility models: Calvet and Fisher (2004), Lux(2008), Lux and Kaizoji (2007) and Lux, Morales-Arias and Satterhoff (2013) all have found certain gains in forecastability of volatility with MSM compared to GARCH-type models. The DJIM seems no exception and also shows complete agreement with more traditional asset classes in terms of its basic statistical features. This, however, casts doubt on whether investment into the stocks represented in the DJIM could provide any safeguard against extreme market gyrations like those observed over the last couple of years.

\section{References}

[1] Andersen TG Bollerslev T (1997) Heterogeneous information arrivals and return volatility dynamics: uncovering the long-run in high frequency returns. Journal of Finance 52:975-1005.

[2] Andreou E, Ghysels E (2002) Detecting multiple breaks in financial market volatility dynamics. Journal of Applied Econometrics 17:579600 . 
[3] Baillie RT, Bollerslev T, Mikkelsen H (1996) Fractionally integrated generalized autoregressive conditional heteroskedasticity. Journal of Econometric 74:3-30

[4] Baillie RT, Morana C (2007) Modeling Long Memory and Structural Breaks in Conditional Variances: an Adaptive FIGARCH Approach. ICER, Working Paper No. 11/2007.

[5] Beine M, Laurent S (2000) Structural change and long memory in volatility: new evidence from daily exchange rates. Working paper, University of Liege.

[6] Ben Nasr A, Boutahar M, Trabelsi A (2010) Fractionally integrated time varying GARCH model. Statistical Methods and Applications 19(3): 399-430.

[7] Bollerslev $\mathrm{T}$ (1986) Generalized autoregressive conditional heteroskedasticity. Journal of Econometrics 31(3), 307-327

[8] Bollerslev T, Mikkelsen HO (1996) Modelling and pricing long memory in stock market volatility. Journal of Econometrics 73:151-184

[9] Bos CS, Franses PH, Ooms M (1999) Long memory and level shifts: re-analyzing inflation rates. Empirical Economics 24,427-449

[10] Brockwell PJ, Dahlhaus R (2004) Generalized Levinson- Durbin and Burg algorithms. Journal of Econometrics 118(1-2), 129-149

[11] Calvet L, Fisher A (2001) Forecasting multifractal volatility, Journal of Econometrics 105, 27-58

[12] Calvet L, Fisher A (2004) Regime switching and the estimation of multifractal processes, Journal of Financial Econometrics 2,49-83

[13] Clark T, West K (2007) Approximately normal tests for equal predictive accuracy in nested models, Journal of Econometrics 138,291-311

[14] Cont R (2001) Empirical Properties of Asset Returns: Stylized Facts and Statistical Issues. Quantitative Finance 1, 223-236

[15] Davidson JEH (2004) Conditional heteroskedasticity models and a new model. Journal of Business and Economic Statistics 22,16-29

[16] Diebold FX (1986) Comment on " Modeling the persistence of conditional variance". by Engle R, Bollerslev T. Econometric Reviews 5:5156

[17] Diebold F X, Mariano R S (1995) Comparing predictive accuracy, Journal of Business and Economic Statistics, 13(3), 253-263. 
[18] Ding Z, Granger CWJ, Engle RF (1993) A long memory property of stock market returns and a new model. Journal of Empirical Finance 1:83-106

[19] Hammoudeh S, Jawadi F, Sarafrazi S (2013) Interactions between conventional and islamic stock markets: a hybrid threshold analysis. Mimeo, Drexel University, PA, U.S.A.

[20] Hansen, PR (2005). A test for superior predictive ability. Journal of Business and Economic Statistics, 23, 365-380.

[21] Hill B (1975) A simple general approach to inference about the tail of a distribution. The Annals of Statistics, 3(5), 1163-1174

[22] Leövey A, Lux T (2012) Parameter estimation and forecasting for multiplicative log-normal cascades, Physical Review, E85, 046114

[23] Liu R, di Matteo T, and Lux T (2007) True and apparent scaling: The proximities of the Markov-Switching Multifractal Model to long-range dependence. Physica A, 383:35-42

[24] Lobato IN, Savin NE (1998) Real and spurious long memory properties of stock market data. Journal of Business and Economic Statistics, $16: 261-268$

[25] Lux T (2008) The Markov-Switching Multifractal Model of asset returns: GMM estimation and linear forecasting of volatility. Journal of Business and Economic Statistics, 26:194-210

[26] Lux T, Kaizoji T (2007) Forecasting volatility and volume in the Tokyo stock market: Long memory, fractality and regime switching. Journal of Economic Dynamics and Control, 31:1808-1843

[27] Lux T, Morales-Arias L, Sattarhoff C (2013) Forecasting daily variations of stock index returns with a multifractal model of realized volatility. Working paper, University of Kiel

[28] Lamoureux CG, Lastrapes WD (1990) Persistence in variance, structural change and the GARCH model. Journal of Business and Economic Statistics, 8:225-34

[29] Mandelbrot B, Fisher A, Calvet LE (1997) A multifractal model of asset returns. Mimeo: Cowles Foundation for Research in Economics.

[30] Martens M, van Dijk D, de Pooter M (2004) Modeling and forecasting SEP 500 volatility: long memory, structural breaks and nonlinearity. Tinbergen Institute Discussion Paper 04-067/4. 
[31] Morana C, Beltratti A (2004) Structural change and long-range dependence in volatility of exchange rates: either, neither or both? Journal of Empirical Finance, 11:629-658

[32] Rapach DE, Strauss, JK (2008) Structural breaks and GARCH models of exchange rate volatility. Journal of Applied Econometrics, 23(1): 6590

[33] Rapach DE, Strauss, JK, Wohar, ME (2008) Forecasting stock return volatility in the presence of structural breaks. In Forecasting in the Presence of Structural Breaks and Model Uncertainty, David E. Rapach and Mark E. Wohar (Eds.), Vol. 3 of Frontiers of Economics and Globalization, Bingley, United Kingdom, Emerald: 381-416 